\title{
INTERRELATION OF INVERSION SYSTEMS IN THE MIDGE CHIRONOMUS INTERTINCTUS (DIPTERA: NEMATOCERA) II. A NONRANDOM ASSOCIATION OF LINKED INVERSIONS
}

\author{
J. MARTIN 1
}

School of Zoology and Genetics, University of Melbourne, Parkville, Victoria, Australia

Received March 17, 1965

$T$ HE three most common inversions in the population of Chironomus (Kiefferulus) intertinctus Skuse at Ocean Grove, Victoria, are tied up in associations of one sort or another. The first paper in this series (Martin 1962) was concerned with the linkage of the Corio inversion, on the third chromosome, with the sex determining region. The present paper deals with a nonrandom association of inversions on the second chromosome, the Lonsdale inversion, with alternative sequences $S t^{L o}$ and $L o$, and the Barwon inversion, with alternative sequences $S t^{B a}$ and $B a$ (MARTIN 1963). This association has been investigated for a number of years in the Ocean Grove population and also in other widely separated populations in an attempt to learn something of its basis.

Many records of similar nonrandom associations appear in the literature for Drosophila (reviewed by Levitan 1958b). The species in which they have been recorded are D. robusta (CARson and Stalker 1949; Levitan 1954 a,b, 1955, 1957, 1958a,b, 1959a,b, 1961a,b, 1964), D. guaramunu (BrNcic 1953; LevitaN and Salzano 1959), D. americana (Blight 1955), D. paramelanica (Stalker 1959, 1960, 1961), D. pavani (Brncic 1961), D. rubida (MATHER 1961, 1963), D. nigromaculata (ToYofuku 1962), D. euronotus (STALKER 1964) and D. subobscura (Krimbas 1964). For other species there appear to be only the cases of Chironomus tentans (Acton 1955), Simulium tuberosum (LANDau 1962) and Simulium vittatum (Pasternak 1964). Also in Tendipes (= Chironomus) decorus, the results of Rothfels and FaIRLIE (1957) show a significant nonrandom association of inversions $A$ and $C$ of chromosome I, such that $S^{A}$ with $S^{C}$ and $A$ with $C$ are favoured. The case of Simulium vittatum must be treated with caution since the significant deviation of inversions IIIL-22 and IIIS-2 is apparently due to deviation of the individual inversions from Hardy-Weinberg expectation, and the significance of this association disappears when the expected values are calculated from marginal totals and are made to add to the observed total.

The majority of these papers simply report the presence of an association in a single population, in some cases not even giving the data upon which their conclusions are based. The only detailed studies are of Drosophila species: $D$.

\footnotetext{
${ }^{1}$ This work was supported by research grants from the U.S. Public Health Service (RG 7212) and from the University of Melbourne.

Genetics 52 : 371-383 August 1965.
} 
robusta, D. paramelanica and D. euronotus. In all cases, the association appears to be related to the geographic distribution of the inversions.

\section{MATERIALS AND METHODS}

The bulk of the material used was collected from Ocean Grove, but other material came from the other localities mentioned in previous work (MARTIN 1962, 1963, 1964). There are also a number of new localities in western Victoria and South Australia for which the inversion frequencies and other data will be published elsewhere. All localities are shown in Figure 1.

Cytological techniques were as outlined by Martin (1962).

\section{RESULTS}

The Lonsdale and Barwon inversions are separated by about 34 bands, and are probably situated on different arms, since meiotic metaphases indicate that chromosome 2 is metacentric. The alternative sequences of both inversions are all quite common in the population at Ocean Grove, although it has been shown that both $L o$ and $B a$ are increasing in frequency (MARTIN 1963), This increase means that the results from different collections cannot be pooled (Table 1). The expected results were calculated on the basis of the marginal totals in order to avoid any possible effects of heterosis in the individual inversions.

In many cases the samples are small and a $x^{2}$-test cannot be legitimately used.

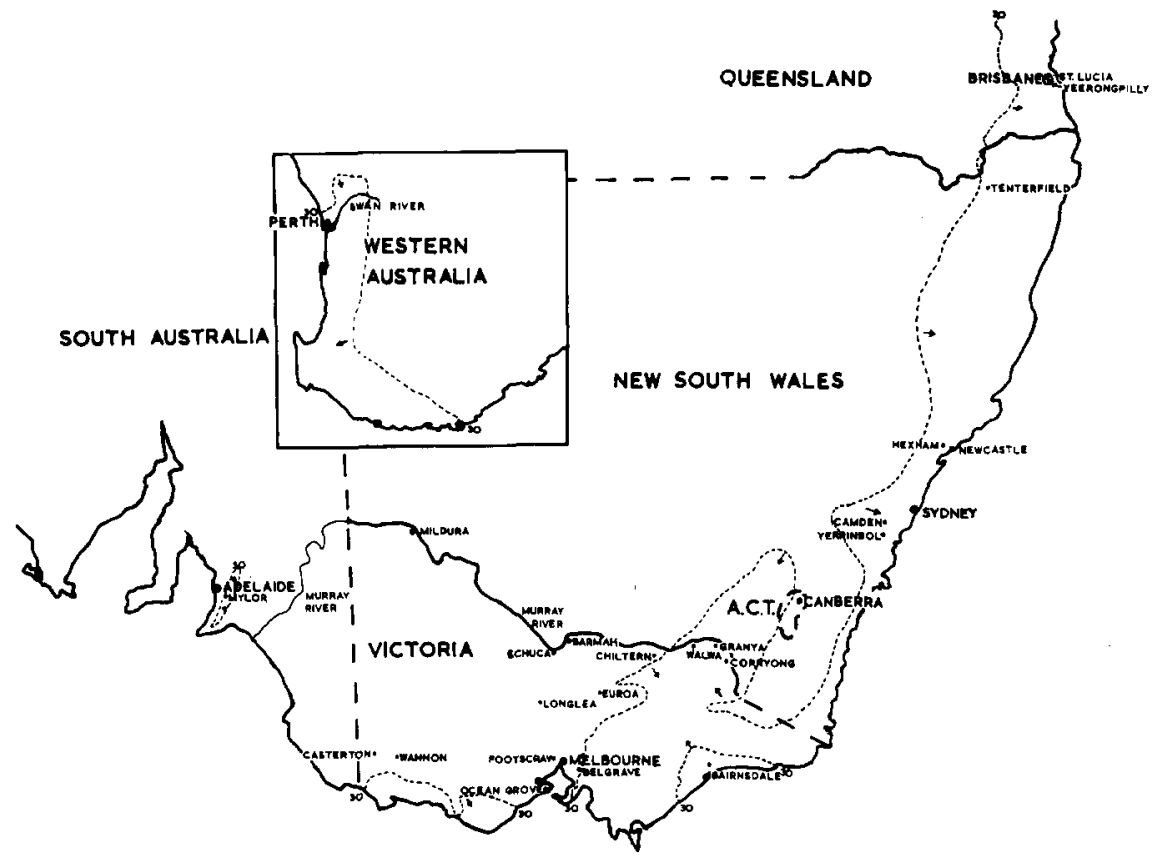

FIGURE 1.-Map of southeastern and southwestern (insert) Australia showing the localities mentioned in the paper. Some of the main cities are also included for reference purposes. The isohyet for 30 inches per year is shown as a fine dotted line, with arrows indicating the direction of increasing rainfall. 


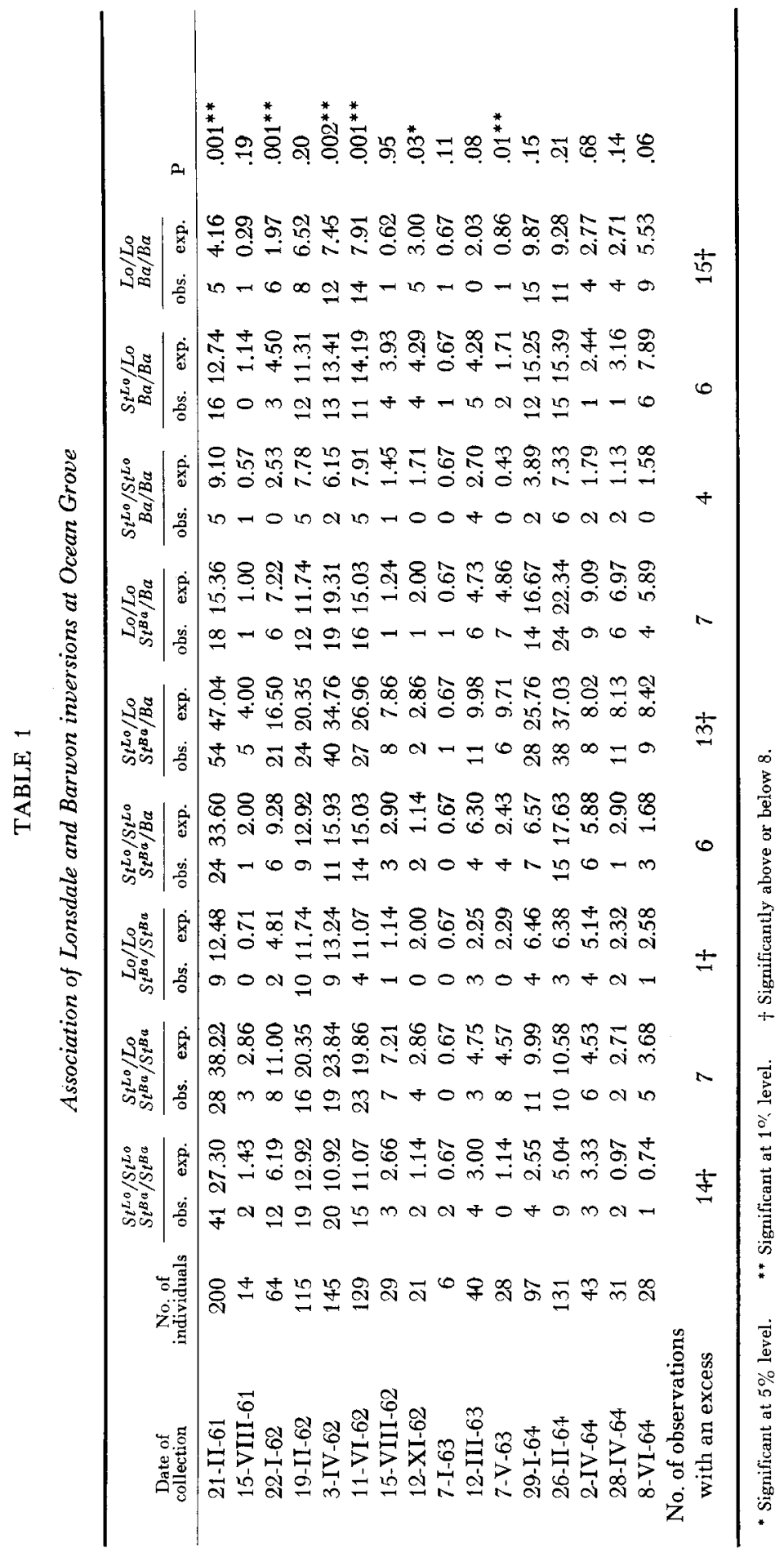


Instead a likelihood ratio test (Fisher 1950) was used, as this is not affected by small expected values. This test uses $\Sigma a_{r} \ln \left(a_{r} / m_{r}\right)$ where $a_{r}$ is the observed, and $m_{r}$ the expected frequency. This sum is distributed in the same manner as $1 / 2 \chi^{2}$, with the same degrees of freedom as the equivalent $\chi^{2}$ test. In addition, the overall pattern of the samples was tested nonparametrically by considering whether the direction of the deviation from expected values for each karyotype is significantly different from $50 \%$, the value that would be expected if there was no consistent association. In Tables 1 to 4 , the cases in which the observed is greater than the expected value are expressed as a fraction of the total number of collections in which there was other than zero deviation.

Despite the small size of many samples, the results show quite clearly that there is a nonrandom association of the Lonsdale and Barwon inversions in this population. The overall pattern is that almost all samples show an excess of $S t^{L o} / S t^{L o}, S t^{B a} / S t^{B a} ; S t^{L o} / L o, S t^{B a} / B a$; and $L o / L o, B a / B a$ individuals, and a deficiency of $L o / L o, S t^{B a} / S t^{B a}$ and $S t^{L o} / S t^{L o}, B a / B a$. The other karyotypes deviate almost equally in either direction. The individual collections that show a significant deviation of observed values from expected values show this same pattern with only one exception, the May 1963 collection, which shows a deficiency of $S t^{L o} / S t^{L o}, S t^{B a} / S t^{B a}$ individuals.

The collections from the Melbourne Botanic Gardens also had to be treated separately (Table 2), because data subsequent to MARTIN 1963 have shown that there is significant heterogeneity in the frequency of the Barwon inversion $\left(x^{2}{ }_{16}=35.9, \mathrm{P}<0.01\right)$. Although all samples from this population are small, they still show evidence of the same association that was present at Ocean Grove. In this poulation, however, the $S t^{L o} / L_{o}, S t^{B a} / B a$ karyotype does not show significantly more than $50 \%$ excesses, the $S t^{L o} / S t^{L o}, S t^{B a} / B a$ karyotype does show a significant number of deficiencies. Once again, the samples that deviate significantly do so in this same pattern.

Table 3 presents data for all other populations except those in which the Hume sequence in the right arm is present. There is no evidence of an overall pattern and, in fact, a significant deficiency of the double heterozygotes and a significant excess of $S t^{L o} / L o, B a / B a$ individuals. However, if the individual populations are taken separately, there is some evidence of the association, although none of the deviations is significant. The population at Yerrinbol, shows the typical pattern of excesses and deficiencies, and the populations at Euroa, Camden, and Hexham, all show a deficiency of $S t^{L o} / S t^{L o}, B a / B a$ individuals. The populations from western Victoria and South Australia show little deviation from expected results, but the majority of the individuals are $L o / L o, B a / B a$. This can be seen in the populations from Adelaide Botanic Gardens, Casterton, and particularly in those from Longlea and Mildura. At Mildura, all the individuals studied were of karyotype $\mathrm{Lo} / \mathrm{Lo}, \mathrm{Ba} / \mathrm{Ba}$.

Populations in which the Hume sequence is present are shown in Table 4. $H u$ includes $B a$ and is separated from the Lonsdale inversion by about 18 bands. The presence of this sequence apparently influences to some extent the association noted in other populations. In most of the populations where $H u$ is present, $S t^{B a}$ 


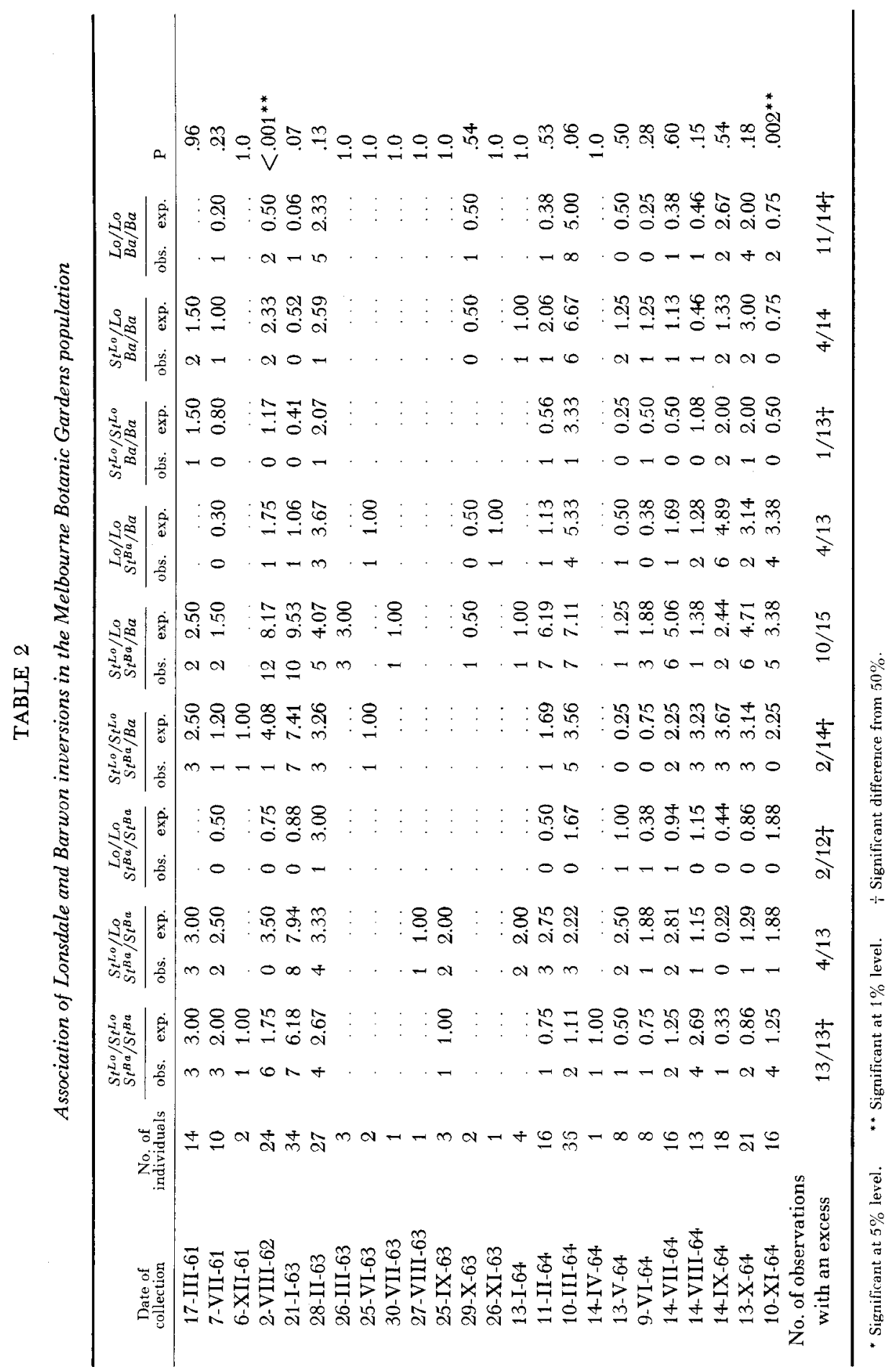




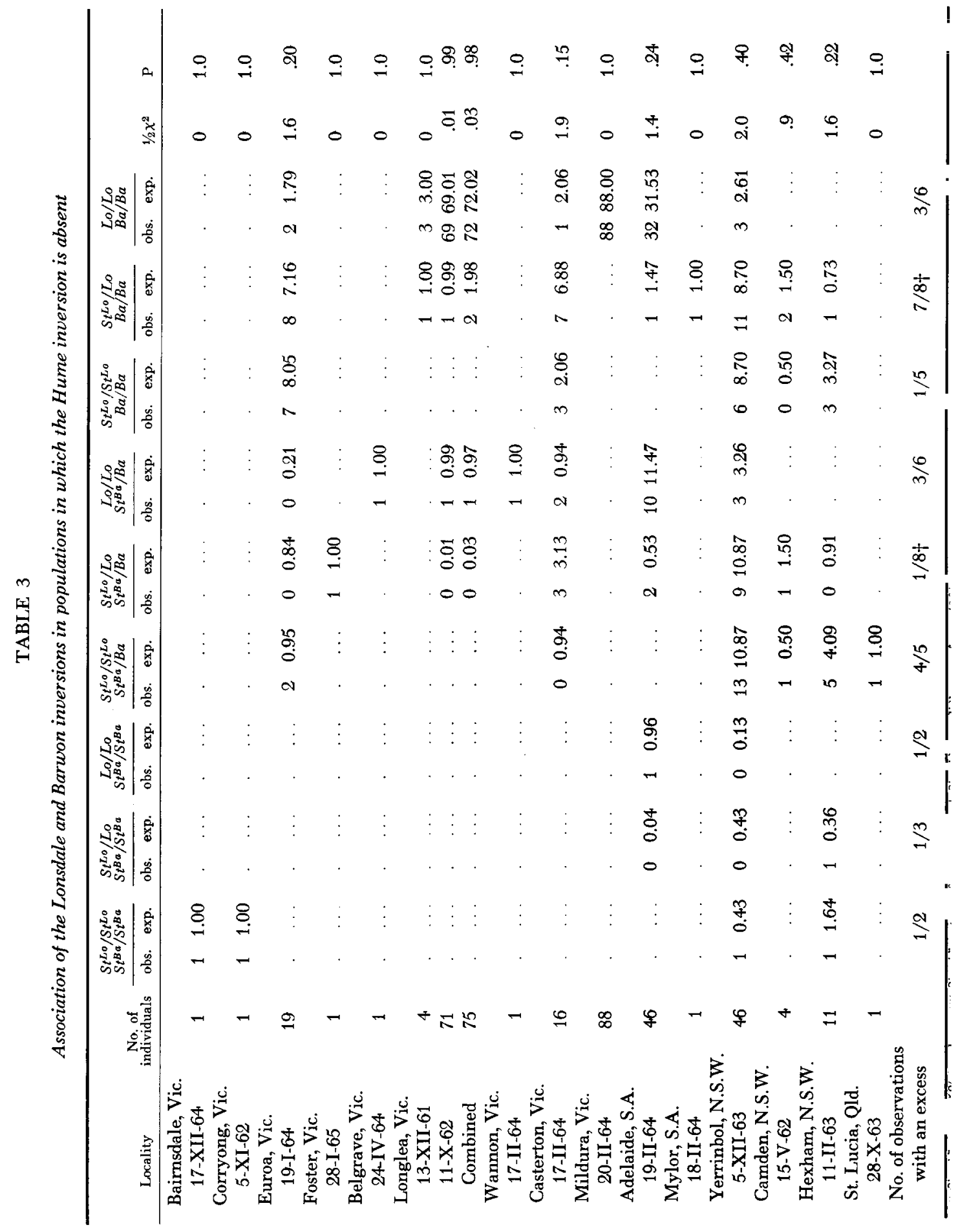




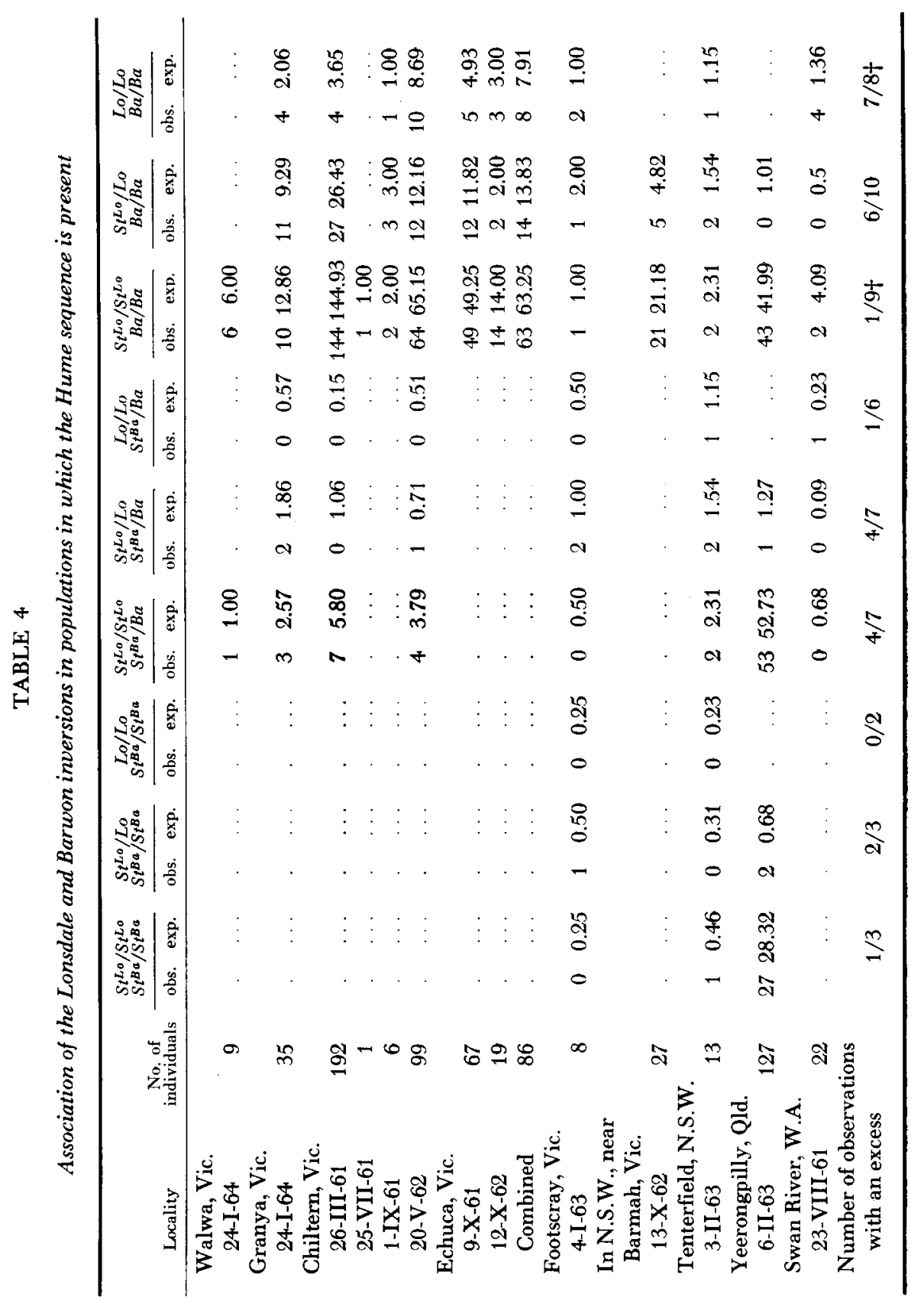




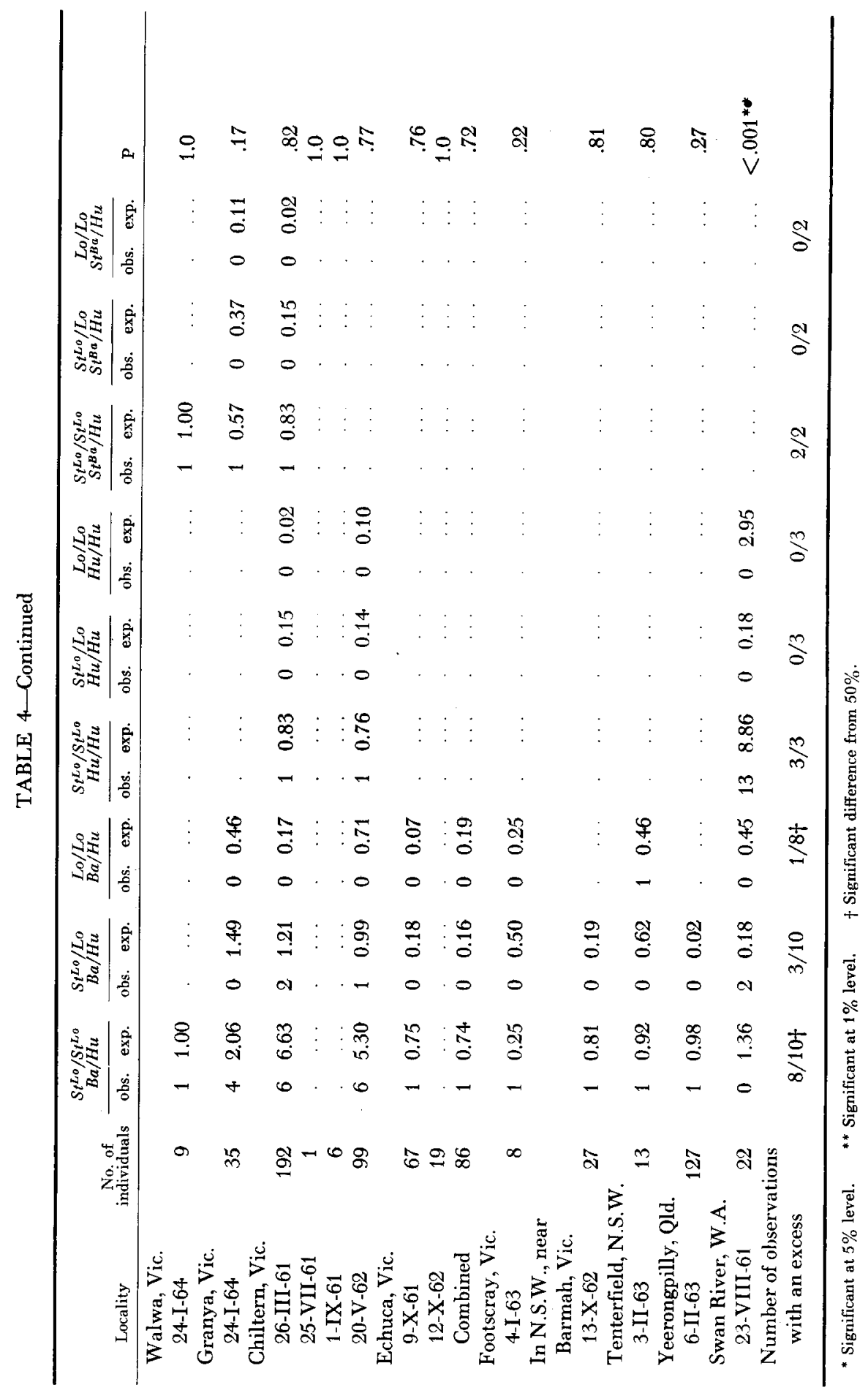


is rare, and $B a$ is the most common sequence. There is still evidence of a deficiency of $S t^{L o} / S t^{L o}, B a / B a$ and an excess of $L o / L o, B a / B a$. However, in only one of the three populations in which $S t^{L o} / S t^{L o}, S t^{B a} / S t^{B a}$ individuals were expected was there an excess. In the other two, including the population at Yeerongpilly where many such individuals were expected, there was a deficiency. On the other hand there was an excess of all combinations of $S t^{L o} / S t^{L o}$ with $H u$. In many cases this was due to the presence of a single individual carrying $H u$, and, since $S t^{L o} /$ $S t^{L o}$ was the most common karyotype of the Lonsdale inversion, it was most likely to occur in this combination. This is not true in the sample from the Swan River where the deviations from expected values are significant.

There is some indication that these associations are more pronounced in females than in males, but this is probably due to the fact that the male samples were usually smaller (MARTIN 1962).

\section{DISCUSSION}

The results from the Ocean Grove population indicate that the Lonsdale and Barwon inversions, like the Corio inversion, are maintained by associations rather than by simple heterosis. This does not mean that the inversions individually do not have adaptive properties. The associations are second-order interactions, the primary interactions being those that occur between the contents of the single inversions (BoDmer and PARsons 1962). It appears that selection is favouring the presence of $S t^{L o}$ with $S t^{B a}$, and also of $L o$ with $B a$, in the same individual, while it is acting against individuals with $S t^{L o}$ and $B a$ or $L o$ and $S t^{B a}$. This has resulted in more chromosomes carrying $S t^{L o}, S t^{B a}$ and $L o, B a$ and fewer carrying $S t^{L o}, B a$ and $L o, S t^{B a}$. This would mean that the two types of double heterozygotes, $S t^{L o} S t^{B a} / L o B a$ and $S t^{L o} B a / L o S t^{B a}$, would not occur in equal frequency. Unlike the investigations with Drosophila, where adults are mated to flies of known karyotype, giving the inversion order on each chromosome, this type of investigation with Chironomus, examining larval chromosomes, makes the determination of chromosome order in double heterozygotes difficult. Such a determination was attempted wherever possible. In many cases, pairing of the homologues between the two inversions is so close that the chromosomes break before the homologues separate. Enough were scored to show that there were more $S t^{L o} S t^{B a} / L o B a(75)$ than $S t^{L o} B a / L o S t^{B a}$ (43), in the total of 293 double heterozygotes. For this purpose the results of the various samples can be pooled, since for random assortment the two types are expected in equal frequency regardless of the inversion frequency. They could not legitimately be pooled for the purpose of calculating whether the proportion of the chromosome types in the double heterozygotes is the same as in the rest of the karyotypes, because the degree of linkage between the inversions may vary with time under the influence of natural selection. Such a calculation on the individual samples, to see if the viability of the two double heterozygotes is the same, is not practicable because so few could be determined.

Parsons (1963a,b) points out that it is not necessary for the viability of the 
two double heterozygotes to be the same, although earlier work on the theory of linkage disequilibria assumed equal viabilities (Lewontin and KoJima 1961; Bodmer and Parsons 1962). Levitan (1955) assumed unequal viabilities of the two types of double heterozygote for the various $D$. robusta associations but his reasons for doing so were not necessarily correct (LevitaN 1958b). Gibson and Thoday (1959, 1962a,b) showed for linked chaeta loci in $D$. melanogaster, a related type of association involving polygenes rather than inversions, that the double heterozygotes did not have equal viabilities, since one type was inviable.

Another question raised in theoretical considerations of linkage disequilibria is whether the associations are due to a cumulative heterozygote advantage (Kimura 1956; Lewontin and Kojima 1960; Lewontin 1964) or to a balanced polygenic complex (Parsons 1963b). Parsons shows that the latter requires a looser linkage for its maintenance. On both models, if one locus is maintained in a balanced polymorphism by heterozygote superiority, then the second will remain polymorphic if linkage is sufficiently close. Heterosis in at least one locus is an essential on the former model (KIMURA 1956), while superiority of at least one of the double heterozygotes is essential for the latter (PARsons 1964). These two requirements may in actual fact be very similar if heterosis at one locus leads to superiority of one of the double heterozygotes. In the present study there is a little evidence of heterosis for the Barwon inversion, but none for the Lonsdale inversion. The Barwon inversion often shows a slight excess of heterozygotes, but the Lonsdale inversion in general shows a slight deficiency. There are no real data to indicate how close the linkage between the inversions might be, but a study of meiosis seems to indicate that crossing over may be restricted to the ends of the chromosome, although it certainly extends at least as far as the actual region of the inversions. The relatively short distance between the inversions, and the fact that they are probably on opposite sides of the centromere, would also suggest that linkage between them is fairly close. Natural selection will be expected to favour closer linkage (Kimura 1956; Bodmer and Parsons 1962), and the intensity of the natural selection reducing recombination will depend on the reproductive potential of the species (Bonmer and PArsons 1962).

Although there does not seem to be the same clear relationship of inversion association and geographic distribution that has been found in Drosophila, there does seem to be some relationship of the sort for the Barwon and Lonsdale inversions. The areas where there is a high frequency of $S t^{L o}$ with $H u$-southern Queensland, eastern New South Wales, north-eastern Victoria, and near Perth in Western Australia - have higher rainfall than areas where there is a high frequency of $L o$ with $B a$-western Victoria and eastern South Australia (Figure 1). There are two samples in which $H u$ was found in lower rainfall areas, at Echuca and Footscray. The presence of $H u$ at Echuca could be explained by migration from populations in the higher rainfall areas up the Murray River. The presence of $\mathrm{Hu}$ at Footscray, however, is rather puzzling, since this is over 100 miles from the other populations in which $H u$ has been found, and $H u$ does not occur at the Melbourne Botanic Gardens, which is only a few miles from Footscray and appears to have similar conditions. 
It appears, then, that the association of $L o$ and $B a$ may be an advantage in lower rainfall areas, possibly enabling the larvae to withstand drying out or higher water temperatures when the pools are at a low level in summer. This is further suggested by the increase in frequency of these two sequences at Ocean Grove, where the pool is now at a generally lower level than when investigations were first begun, due to a large dam cutting off one of the major sources of run-off filling the pool. During summer this pool now comes much closer to drying out, and the mud surface temperature is higher. The association of $S t^{L t}$ and $H u$ on the other hand may be an advantage in the higher rainfall areas. There is no indication as to what the advantage of $S t^{L O}$ and $S t^{B a}$ may be in the rather restricted area where they are both common, in south central Victoria. Since the advantage of $L o$ with $B a$ seems to be maintained throughout the area studied, it may be that it is the selective advantage of these two sequences occurring together that is the real basis of the observed association.

The single heterozygotes in general show no significant deviation from expectation, as would be expected if the advantageous combination was roughly counterbalanced by the disadvantageous combination. This is not uniform in all populations; $S t^{L O} / L o, B a / B a$ is significantly in excess in New South Wales and western Victoria (Table 3), and $S t^{L o} / S t^{L o}, S t^{B a} / B a$ is significantly deficient at the Melbourne Botanic Gardens (Table 2). The double heterozygotes also vary between populations, only showing a significant excess at Ocean Grove. These effects are most likely attributable to the different karyotypes having different adaptive values in the various populations.

In general, then, the nonrandom association reported here for Chironomus appears to be similar in nature to the well-studied associations in Drosophila robusta, D. paramelanica and D. euronotus.

The author wishes to express his appreciation to Professor M. J. D. White for his assistance throughout this work and for his criticisms of the manuscript, to Dr. P. A. PArsons, who also read the manuscript, and to Dr. R. E. BLACKITH, who suggested the statistical tests.

\section{SUMMARY}

The second chromosome inversions Barwon and Lonsdale show evidence of a nonrandom association, apparently favouring the occurrence together of the two Standard sequences and also of $\mathrm{Lo}$ with $\mathrm{Ba}$. Where $\mathrm{Hu}$ is present, the association of the two Standard sequences apparently breaks down and the association of $\mathrm{St}^{L o}$ with $\mathrm{Hu}$ is favoured instead. The underlying basis of the association may be in withstanding higher water temperatures in summer. $L o$ and $B a$ together are at an advantage in such conditions, while $S t^{L o}$ and $H u$ together are an advantage in higher rainfall areas. The reason for the advantage of $S t^{L O}$ and $S t^{B a}$ where they occur together in relatively high frequencies, is unknown.

\section{LITERATURE CITED}

Acron, A. B., 1955 Selective values of chromosome inversions in Chironomus. Proc. Roy. Phy's. Soc. Edinburgh 24: 10-14. 
Blight, W. C., 1955 A cytological study of linear populations of Drosophila americana near St. Louis, Mo. Ph.D. thesis, Washington University, St. Louis. (Quoted in Levitan 1958b).

Bodmer, W. F., and P. A. Parsons, 1962 Linkage and recombination in evolution. Advan. Genet. 11: 1-100.

BrNcic, D., 1953 Chromosomal variation in natural populations of D. guaramunu. Z. Ind. Abst. Vererb. 85: 1-11. — 1961 Nonrandom association of inversions in Drosophila pavani. Genetics 46: 401-406.

Carson, H. L., and H. D. Stalker, 1949 Seasonal variation in gene arrangement frequencies over a three year period in Drosophila robusta Sturtevant. Evolution 3: 322-329.

Fisher, R. A., 1950 The significance of deviations from expectation in a Poisson series. Bismetrics 6: 17-24.

Gibson, J. B., and J. M. Thoday, 1959 Recombinational lethals in a polymorphic population. Nature 184: $1593-1594$. — 1962a Effects of disruptive selection. VI. A second chromosome polymorphism. Heredity 17: 1-26. - 1962b An apparent 20 map unit position effect. Nature 196: 661-662.

Krmura, M., 1956 A model of a genetic system which leads to closer linkage by natural selection. Evolution 10: 278-287.

Krimbas, C. B., 1964 The genetics of Drosophila subobscura populations. II. Inversion polymorphism in a population from Holland. Z. Vererb. 95: 125-128.

LANDAU, R., 1962 Four forms of Simulium tuberosum (Lundstr.) in Southern Ontario: a salivary gland chromosome study. Can. J. Zool. 40: 921-939.

Levitan, M., 1954a Position effects in natural populations. Am. Naturalist 88 : 419-423. $1954 \mathrm{~b}$ Additional evidence of position effects in natural populations. Genetics 39: 979. 1955 Studies of linkage in populations. I. Associations of second chromosome inversions in Drosophila robusta. Evolution 9: 62-74. — 1957 Natural selection for linked gene arrangements. (Abstr.) Anat. Record 127: 430. _ 1958a Studies in linkage in populations. II. Recombination between linked inversions of $D$. robusta. Genetics 43: 620 633. — 1958b Non-random associations of inversions. Cold Spring Harbor Symp. Quant. Biol. 23: 251-268. — 1959a A nonrandom association of inversions correlated with their geographic distribution. (Abstr.) Genetics 44: 522. _ 1959b Non-random associations of linked inversions in a third locality. (Abstr.) Anat. Record 133: 403.

1961a A complex X-chromosome linkage association. (Abstr.) Anat. Record 139: 312. — 1961b Proof of an adaptive linkage association. Science 134: 1617-1619.

1964 Geographic and sex factors in an autosomal linkage disequilibrium. J. Am. Med. Women's Assoc. 19: 320-329.

Levitan, M., and F. M. Salzano, 1959 Studies of linkage in populations: III. An association of linked inversions in Drosophila guaramunu. Heredity 13: 243-248.

Lewontin, R. C., 1964 The interaction of selsction and linkage. I. General considerations; heterotic models. Genetics 49: 49-67.

Lewontin, R. C., and K. Ko.rims, 1960 The evolutionary dynamics of complex polymorphisms. Evolution 14: 458-472.

Martin, J., 1962 Interrelation of inversion systems in the midge Chironomus intertinctus (Diptera: Nematocera), I. A sex-linked inversion. Australian J. Biol. Sci. 15: 666-673. 1963 The cytology and larval morphology of the Victorian representatives of the subgenus Kiefferulus of the genus Chironomus (Diptera: Nematocera). Australian J. Zool. 11: 301-322. — 1964. Morphological differences between Chironomus intertinctus Skuse and $C$. paratinctus, sp. nov., with descriptions and a key to the subgenus Kiefferulus (Diptera: Nematocera). Australian J. Zool. 12: 279-287.

MAther, W. B., 1961 Chromosomal polymorphism in Drosophila rubida Mather. Genetics 46: 
799-810. _- 1963 Patterns of chromosomal polymorphism in Drosophila rubida. Am. Naturalist 97 : $59-64$.

Parsons, P. A., 1963a Complex polymorphisms where coupling and repulsion double heterozygote viabilities differ. Heredity 18: 369-374. —— 1963b Polymorphism and the balanced polygenic combination. Evolution 17: 564-574. - 1964 Polymorphism and the balanced polygenic complex-a comment. Evolution 18: 512.

Pasternak, J., 1964 Chromosome polymorphism in the blackfly Simulium vittatum (Zett.). Can. J. Zool. 42: 135-158.

Rothrels, K. H., and W. FaIrLIE, 1957 The nonrandom distribution of inversion breaks in the midge Tendipes decorus. Can. J. Zool. 35 : 221-263.

STALKER, H. D., 1959 Nonrandom associations of inversions in chromosomes of wild Drosophila paramelanica. (Abstr.) Genetics 44: 538. — 1960 Chromosomal polymorphism in Drosophila paramelanica Patterson. Genetics 45: 95-114. _— 1961 The genetic systems modifying meiotic drive in Drosophila paramelanica. Genetics 46: 177-202. — 1964 Chromosomal polymorphism in Drosophila euronotus. Genetics 49 : 669-687.

Toyofuku, Y., 1962 Drosophila survey of Hokkaido. XVII. A study of chromosomal polymorphism in natural populations of Drosophila nigromaculata. Japan. J. Genet. 37: 291-301. 